\title{
Cross Strip Anode Imaging Readouts for Microchannel Plate Detectors
}

\author{
O.H.W. Siegmund, A. Tremsin, J.V. Vallerga and J. Hull \\ Space Sciences Laboratory \\ University of California, Berkeley, CA 94720
}

\begin{abstract}
We have developed a novel microchannel plate readout scheme, the cross strip anode. The cross strip anode has a coarse $(0.5 \mathrm{~mm})$ multilayer metal and ceramic cross strip pattern that encodes event positions by direct sensing of the charge on each strip and subsequent determination of the charge cloud centroid for each event. Event position encoding is accomplished with chip level preamplifiers on the anode, subsequent analog to digital conversion of individual strip charge values and a software centroid determination. We find that the spatial resolution $(<7 \mu \mathrm{m})$ is sufficient to resolve $12 \mu \mathrm{m}$ microchannel plate pores. The cross strip anode can achieve this resolution while using low MCP gain $\left(5 \times 10^{6}\right)$, thus increasing the local counting rate capacity and overall lifetime of the microchannel plate detector system. The image linearity is good enough $(<5 \mu \mathrm{m})$ to enable distortions in the microchannel plate hexagonal boundaries to be seen. We also discuss plans for custom chip electronics development so that encoding may be accomplished at photon counting rates $>1$ $\mathrm{MHz}$ and with low power consumption ( 2W).
\end{abstract}

\section{INTRODUCTION}

There are a large number of imaging readout schemes that have been used for microchannel plate (MCP) detector systems [1]. Each has its merits, but none have yet achieved a distortion free system with position resolution sufficient to resolve MCP pores, which also accommodates large formats, and has small volume, low power encoding electronics. We have taken a significant step towards this goal in the form of the cross strip anode readout scheme.

The cross strip (CS) anode concept [2] has some of the design attributes of ar current MCP readout scheme, the cross delay line (XDL) anode [3]. CS anode designs have two orthogonal sets of conductive strips, one for $\mathrm{X}$, and one for $\mathrm{Y}$ position encoding. The structure of CS anodes is relatively coarse $(\sim 0.5 \mathrm{~mm}$ period) and can be fabricated with the operational robustness already proven with XDL anodes. The two strip sets are separate layers in a multi-layer structure and collect the event signal from the MCP's with about equal charge sharing. The CS anode scheme uses the charge detected on each strip to determine the charge cloud centroid for each axis. To ensure that an accurate event centroid can be determined the charge cloud is matched to the anode period so that charge is collected on several neighboring fingers. The charge collected on the strips is sensed by charge sensitive amplifiers connected individually to each strip.
To encode the event position for each axis the strip which corresponds to the center peak of the charge cloud, must be determined. Then the calculation of the charge cloud centroid to a small fraction of the strip width must be accomplished. This coarse and fine position information is combined to provide the $\mathrm{X}$ and $\mathrm{Y}$ event centroid.

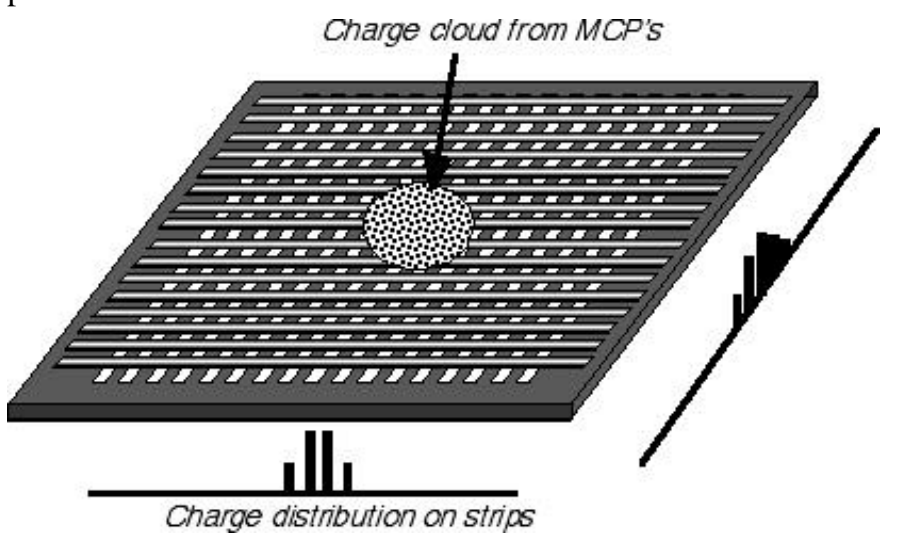

Figure 1. Schematic of the cross strip anode showing the MCP charge cloud, and charge distribution on the cross strips.

\section{CROSS STRIP ANODE DESIGN}

The CS anode construction begins with an alumina, substrate which has good high frequency propagation properties, and withstands high temperatures with low gas evolution. The first set of conductive strips, interconnecting leads and the ground plane are applied and fired onto the alumina substrate using copper or silver. The set of strips is approximately $0.4 \mathrm{~mm}$ wide with $0.5 \mathrm{~mm}$ period, and as long as necessary for the image format size. A set of ceramic insulating strips is then applied in the orthogonal direction to the base strips such that $50 \%$ of the bottom layer is left exposed. An identical set of conductive strips is placed on top of the ceramic strips as a ground plane to prevent crosstalk between the sensing strips. Another identical ceramic and conductor layer are then added above the ground strips. Between each layer application the anode is fired at $\sim 900^{\circ} \mathrm{C}$. The strips of the lower layer and the uppermost layer are connected to the preamplifier chip(s) by means of conductor "fan-ins" on the base substrate. After the preamplifier chips have been bonded to the anode substrate final connections from the fan-in to the preamplifier channels are made by gold wire bump bonds. 


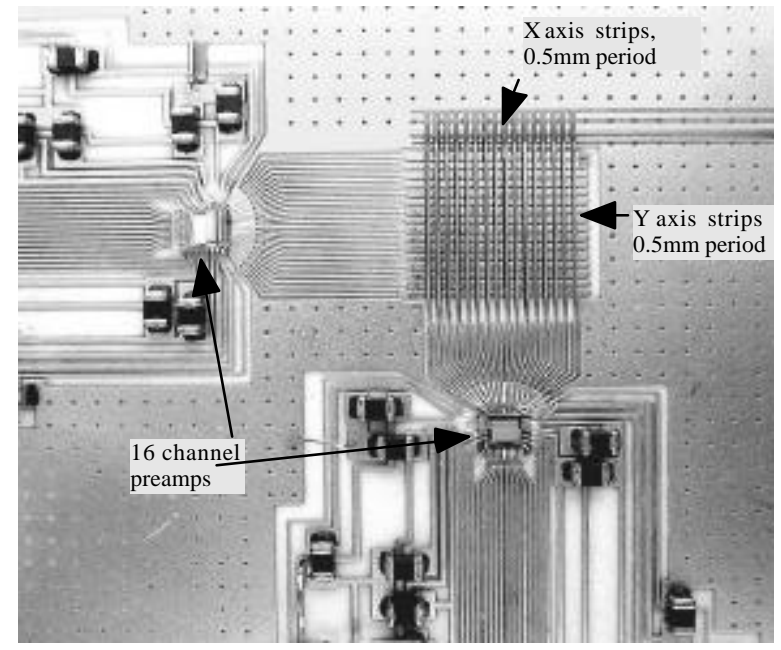

Figure 2: Photograph of the CS test anode showing the cross strips, preamplifier chips and interconnect structures.

The anodes that we have made for initial testing have $16 \mathrm{x}$ 16 strips on a $8 \mathrm{~mm} \times 8 \mathrm{~mm}$ format. This was done to conveniently match existing 16 channel preamplifier chips that were available. Connections for power and the preamplifier outputs were made with vacuum compatible " $D$ " type connectors mounted on a vacuum flange and directly soldered to the anode substrate.

\section{DETECTOR CONFIGURATION}

The detector configuration for the CS anode testing (Figure. 3) is essentially the same as XDL detectors. A brazed metal and alumina ceramic housing contains the microchannel plate stack. In this case an MCP pair with $12 \mu \mathrm{m}$ pores and 80:1 length to diameter ratio was used in a back to back stack. This was positioned $\sim 3 \mathrm{~mm}$ above the CS anode to allow the charge cloud to spread over 5 strips.

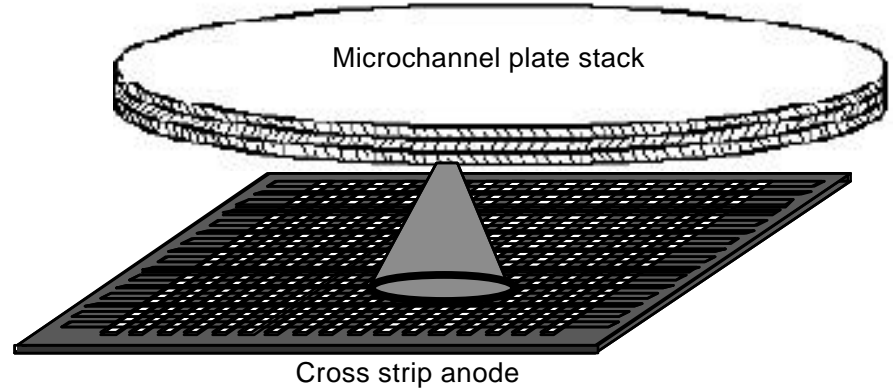

Figure 3: Configuration of MCP's and CS test anode. For most tests two MCP's with $12 \mu \mathrm{m}$ pores and 80:1 length to diameter ratio were used, positioned $\sim 3 \mathrm{~mm}$ above the anode.

The potential applied between the MCP output and the CS anode can be adjusted to achieve the optimal charge cloud size. The multichannel preamplifier chips are mounted directly to the front of the anode as shown previously, but all the downstream electronics is mounted externally.

\section{ELECTRONIC CONFIGURATION}

The overall electronics processing chain for encoding event positions with the CS anode is shown in Figure 4. Each strip on the CS anode must be connected to an individual amplifier. This requires a significantly larger number of amplification channels (about 48 per axis for a $24 \mathrm{~mm}$ format) than wedge and strip (3 amplifiers)[1] or cross delay line (4 amplifiers) readout systems. However, improved designs with better chip level amplifier reliability, power consumption and packaging have made the use of multi-amplifier chips of this type routine. The issue of bonding and connecting multiple chips to an anode is also not a particularly difficult task with current wire bonding methods.

The preamplifier chip design (ICD2) that we have used was developed for microstrip detectors by Lawrence Berkeley National Laboratory (LBNL)[4]. This chip is $\sim 2 \mathrm{~mm} \times 2 \mathrm{~mm}$ in bare die form and provides sixteen parallel channels with a gain of $1.9 \times 10^{6} \mathrm{e}^{-} \mathrm{V}^{-1}$ and noise of $<500 \mathrm{e}^{-} \mathrm{RMS}$ with low input capacitance load $(<2 \mathrm{pF})$. The unipolar time constant $(\mathrm{T}=20 \mathrm{~ns}$ peaking time) is short and the power dissipation is low (10.5 $\mathrm{mW} / \mathrm{channel}$ ). For our anode the strip capacitances are in the 2 $-3 \mathrm{pF}$ range, and the charge signals from the MCP's have 2 ns width. So the ICD2 is well matched to our application, and we expect peak strip signals of $<1$ volt at MCP gains of $5 \times 10^{6}$.

The preamplified signals are subsequently passed through the vacuum flange on "D" type connectors to external shaping amplifier circuits and 50? signal drivers. Here we also apply a discrimination based on the amplitude of the signals from the output face of the MCP stack to reject low amplitude noise. The shaped strip signals are digitized with two 16 channel CAMAC peak sensing 12 bit ADC's (Phillips 7164). The data from the ADC's are transferred from the CAMAC crate to a SCSI interface in a Macintosh computer. Each event is a now set of digitized strip signal amplitudes. Using National Instruments Labview software routines, we determine the strip at which the peak charge occurs for $\mathrm{X}$ and $\mathrm{Y}$. Then the centroid of the four highest neighboring strip signals in each axis is calculated to provide the interpolated fine $(\mathrm{x}, \mathrm{y})$ position for each valid event. This software will not permit the high speed event processing which could be realized, but it allows performance measurements for the detector system imaging to be made.

Other issues that are important include compensation for small gain variations in the individual amplifier chains, DC signal offsets, and accommodation for signal crosstalk between strips. The signal crosstalk between the two axes is small $(<0.1 \%)$, but the DC offset is not negligible. Therefore we apply a threshold to the strip data before centroid calculation. Small gain variations between amplifier chains are measured and used to implement weighting factors for the individual strip signals in the centroid calculations. The final $\mathrm{X}$ and $\mathrm{Y}$ event coordinates are stored as an event list that can then be binned and displayed as an accumulated image. 


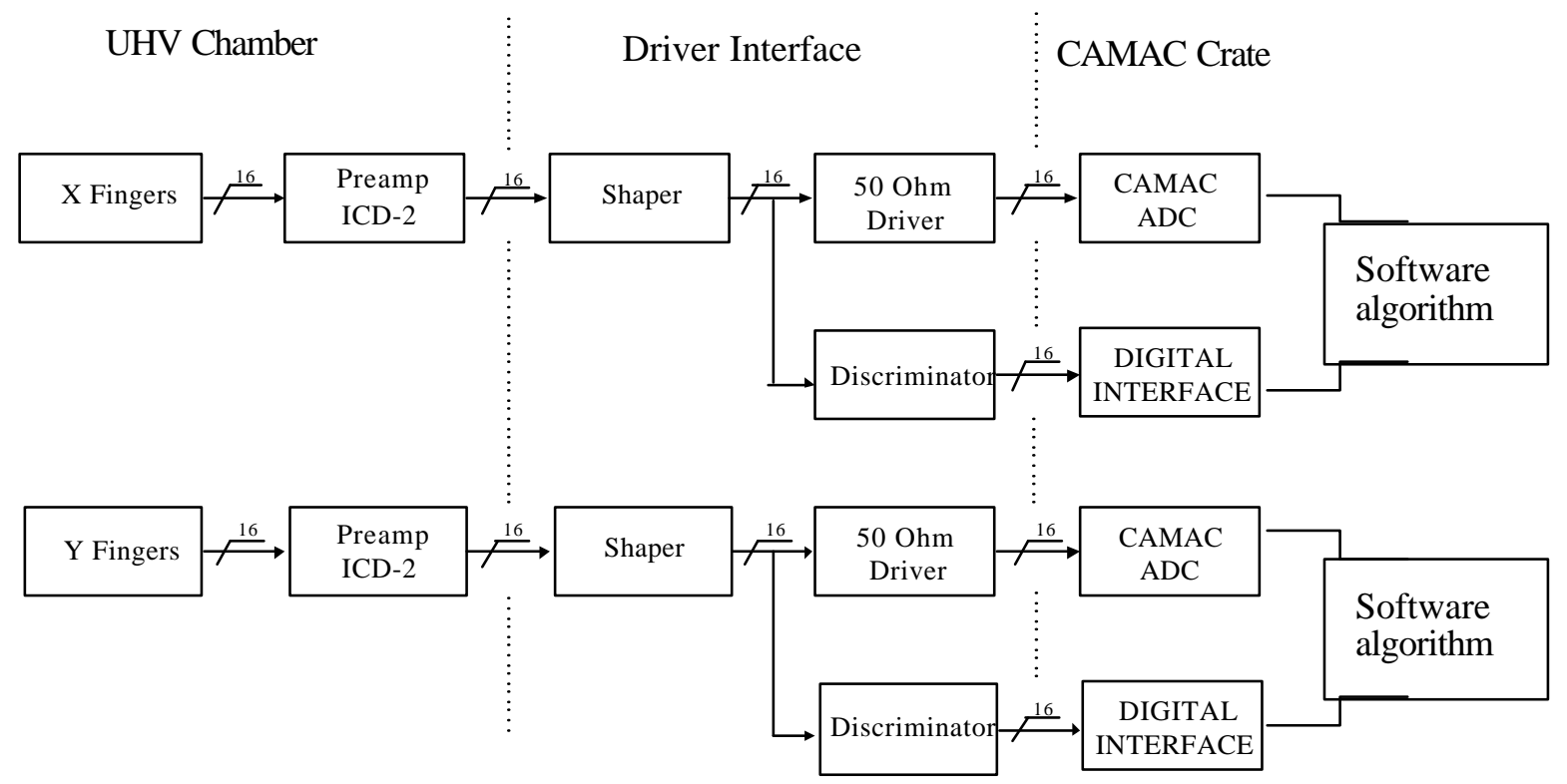

Figure 4. Schematic of the CS anode position encoding electronics. The ICD2 preamplifier die is mounted directly on the CS anode surface, while the shaping amplifier, driver and discriminator board is about $1 \mathrm{~m}$ away, and connects via a $2 \mathrm{~m}$ cable to the CAMAC ADC. The 12 bit ADC signals are aquired via a SCSI interface to a Macintosh computer, then processed by a custom National Instruments Labview routine to derive event centroid positions.

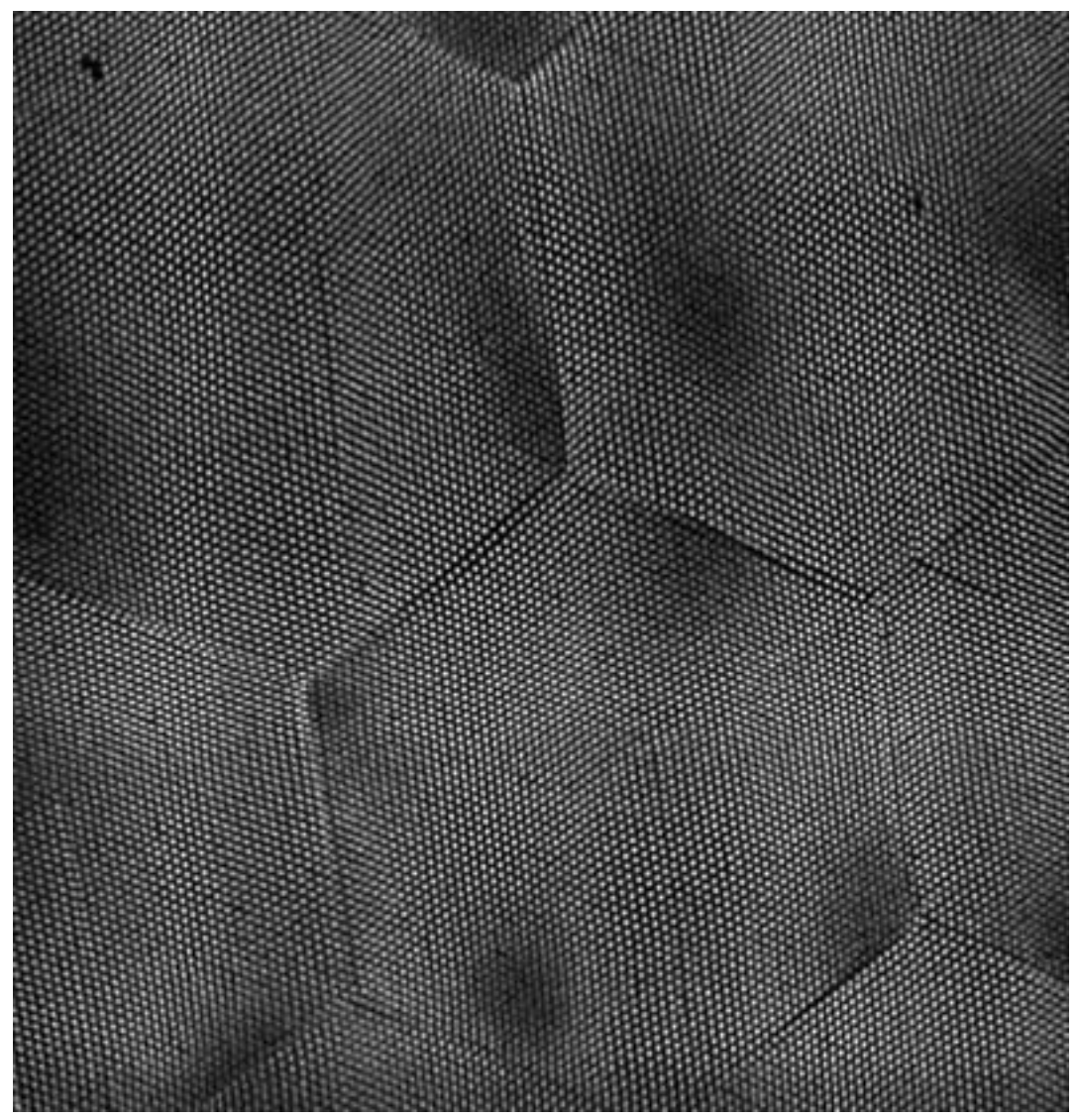

Figure 5. Image of a $2 \mathrm{~mm} \times 2 \mathrm{~mm}$ section of the cross strip MCP detector showing the MCP hexagonal multi-fibers, pores and patches due to Moire interference. MCP gain $5.5 \times 10^{6}$, MCP pair with $12.5 \mu \mathrm{m}$ pores, and 80:1 pore length/diameter ratio. 


\section{Detector Test Results}

\section{A. Resolution Tests}

For our initial testing we placed a mask directly onto the MCP front surface. This mask contains $10 \mu \mathrm{m}$ diameter pinholes spaced at $2 \mathrm{~mm}$ intervals in both axes. The accumulated images at MCP gain of $4 \times 10^{6}$ showed the regular array of pinhole images. At the $15 \mu \mathrm{m}$ electronic binning level the pinhole images were only one bin wide. Since we have sufficient accuracy in the centroid calculation (12 bit digitization per strip charge) we can change the electronic binning accuracy to suit our image sampling requirements. It is possible to calculate the event centroid to better than 1 part in 1000 of the strip period, or $0.5 \mu \mathrm{m}$, although that would be excessive for these measurements. Changing to a $7 \mu \mathrm{m}$ position sample binning the pinhole images were still only 1 or 2 bins wide thus indicating resolution similar to the MCP pore dimensions.

At this point we removed the pinhole mask to determine the flat field characteristics of the CS anode readout scheme.

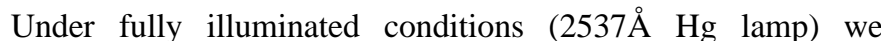
obtained the image shown in Figure 5 for a $2 \mathrm{~mm} \times 2 \mathrm{~mm}$ section of the detector with an electronic binning level of $2 \mu \mathrm{m}$. Due to the limitations of the software, counting rates were limited to less than $1 \mathrm{kHz}$, so that a full detector image with good statistics would take a prohibitively long time. The image clearly shows the MCP pores and the hexagonal multi-fiber boundaries.

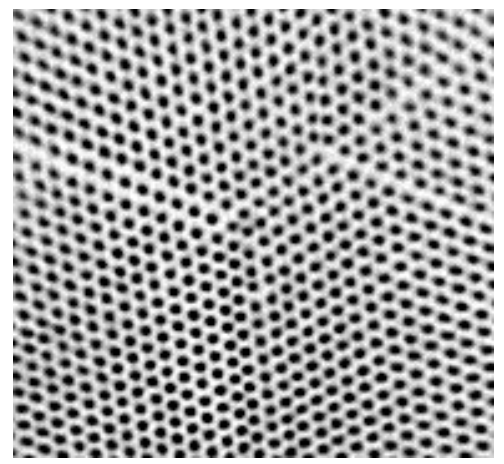

Figure 6: Image (inverted) of a small section of Figure 5 including several MCP multi-fiber boundaries.

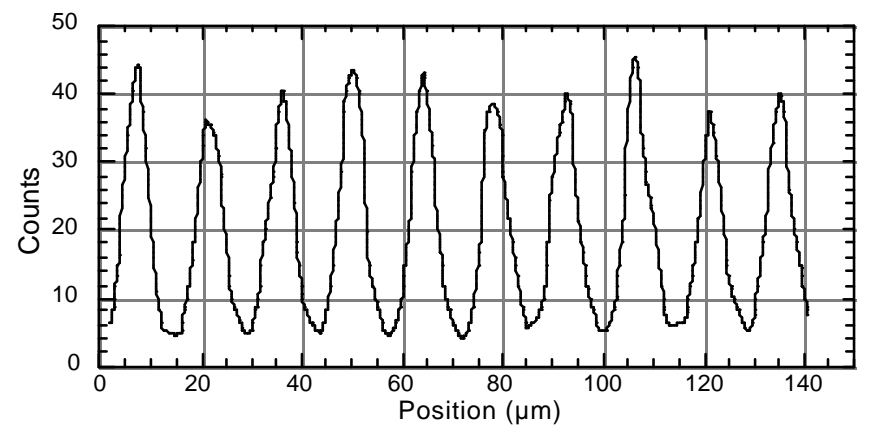

Figure 7: Histogram for a slice through some pore images shown in Figure 6 showing about $7 \mu \mathrm{m} \pm 1 \mu \mathrm{m}$ FWHM for the pore images.
Other features that are clearly visible include dead pores, shear and distortion at multi-fiber boundaries and patches of low intensity in the image. Many of these effects can be attributed to the manufacture of the MCP's themselves. It is difficult to put a metric on the contributions of the readout system itself since there are no fixed patterns with which to assess this. Histograms of slices through pore images, such as shown in Figure 6, and Figure 7, allow some assessments to be made. The intensity modulation of $>80 \%$ and peak width of pores in Figure 7 clearly shows that the CS anode resolution is of the order $7 \mu \mathrm{m}$ full width at half maximum (FWHM). Since the pore open area ratio for these MCP's is about $70 \%$ an optical pore image would have a flat topped intensity histogram, with $12.5 \mu \mathrm{m}$ wide tops and $1 \mu \mathrm{m}$ to $2 \mu \mathrm{m}$ valleys. Due to the nature of the centroid position encoding of the CS anode we are determining the center positions of the MCP pores of the front MCP of the stack. Positions within the pore diameter are not meaningful since the output charge cloud does not depend on the input position at the top of the pore of the front MCP. Thus the FWHM of the pore images are attributable to the accuracy of the image readout system and the statistical variations of the MCP charge cloud position. The latter are small, $\sim 1 \mu \mathrm{m}$, at MCP gain of $4 \times 10^{6}$ assuming a $2.5 \mathrm{~mm}$ charge cloud.

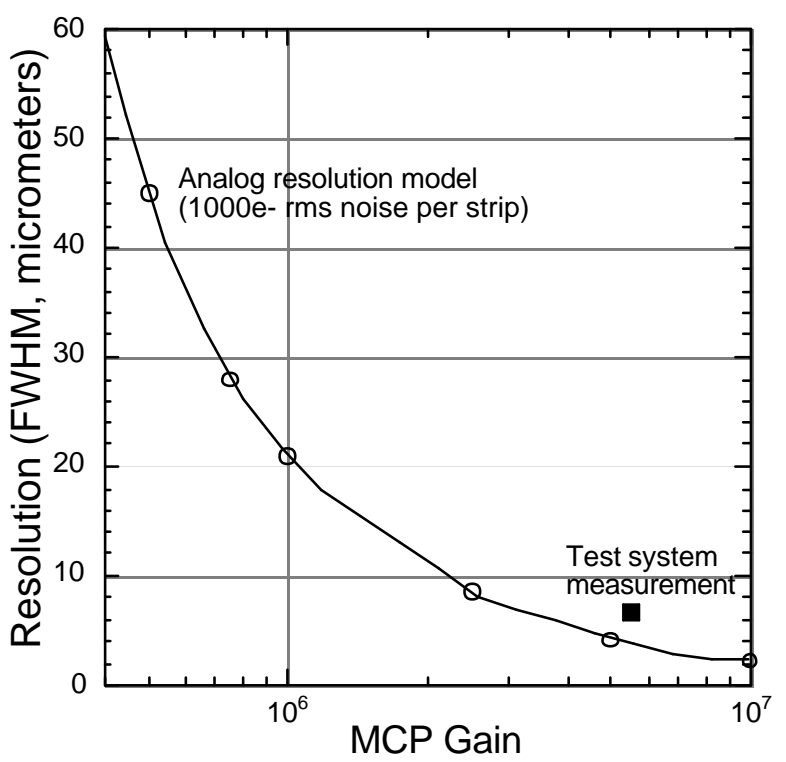

Figure 8: Comparison of the calculated position resolution assuming 1000 e- RMS noise for a $0.5 \mathrm{~mm}$ strip period and a 5 strip centroid algorithm with the measured resolution data.

We have calculated the expected position resolution (Figure 8) of the CS anode system assuming a 1000 e- RMS noise per strip, a five strip centroid calculation, and a $0.5 \mathrm{~mm}$ strip period. We have omitted the ADC bit digitization error, although at 12 bits it will less than to the $1000 \mathrm{e}$ - amplifier noise value. The calculation indicates $\sim 5 \mu \mathrm{m}$ resolution at $5 \times 10^{6}$ gain which is very close to our experimental result. Adding the $\mathrm{ADC}$ error in quadrature we expect about $6-7 \mu \mathrm{m}$ resolution at $5 \times 10^{6}$ which is essentially our experimental result. However, the practical resolution is actually the MCP pore size of $12 \mu \mathrm{m}$. Therefore, to obtain better spatial resolution we must use MCP's with smaller pores. Indeed 
MCP's with $5 \mu \mathrm{m}$ pores are available and this is the next logical test we plan to do.

\section{B. Image Linearity}

As pointed out earlier there are a many image effects visible with resolution at the MCP pore level. The most obvious is the hexagonal multi-fiber boundaries. A histogram of pore images (Figure 9) across a multi-fiber boundary clearly shows that the pore spacing changes by 3 to $4 \mu \mathrm{m}$ at some of these boundaries. A close examination of the image in Figure 5 indicates that there is a faint second multi-fiber pattern offset by about half the width of a multi-fiber. This is most likely the CS anode resolving the multi-fiber on both the top and bottom MCP's of the stack. Looking at in Figure 5 at a glancing angle one can see the linearity of the rows of pores, and the dislocations at multi-fiber boundaries. There may also be a small "wave" distortion that is a residual of the 5 strip centroid algorithm.

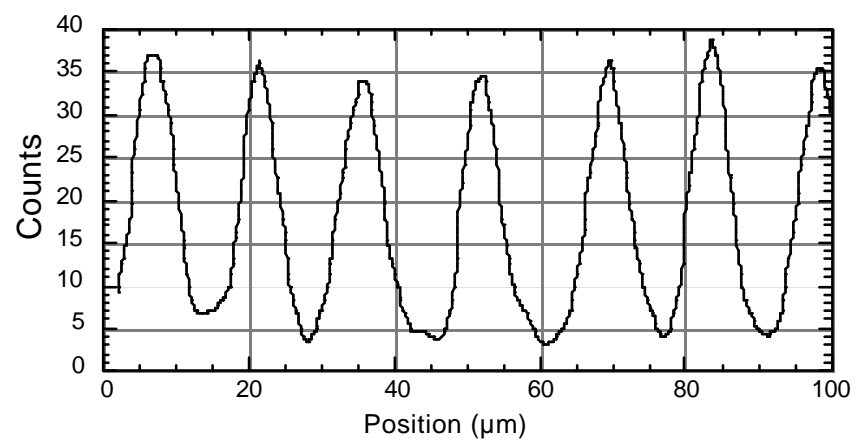

Figure 9: Histogram for a slice through pore images at a multifiber boundary shown on the upper left in Figure 6 . The pore spacing increases from $\sim 15 \mu \mathrm{m}$ to $\sim 18 \mu \mathrm{m}$ at the boundary.

Another obvious feature are the darker patches in the image where the pore image definition also seems poorer. We believe this to be related to Moire interference between the very similar pore hexagonal packing arrangement of the two MCP's. Our earlier evaluations [5] of the Moire interference effect showed that many different kinds of image intensity modulation can occur over a range of spatial frequencies depending on the rotational angle between the hexagonal multifibers of the two MCP's. Some of the effects seen give zones of interference very similar to those seen in Figure 5 when the hexagonal multifibers are almost aligned. This seems to be so since the fainter multifiber pattern is aligned but slightly offset from the stronger multifiber pattern.

\section{CONCLUSIONS}

We have shown that the CS anode can provide spatial resolving characteristics that are better than most other electronic imaging readout systems [1,6]. Only centroiding intensified CCD's/CID's have shown comparable results [1,7] but have event rate limitations particularly for large detector formats. However, due to its inherently fast signal propagation speed, the CS anode, equipped with the appropriate encoding electronics (fast ADC's), should accommodate $>1 \mathrm{MHz}$ event processing rates with time tagging of a few 10's of picoseconds. Since the capacitive load increases linearly with the anode size, formats of $>50 \mathrm{~mm}$ should be possible without significant degradation of the electronic performance. Anode structures of this size and type have already been built for XDL's. The MCP gain required for CS anodes is a factor of four lower than XDL's, allowing higher MCP local counting rates (1000 events pore $\left.\mathrm{sec}^{-1}\right)$ to be supported, while also extending the overall MCP lifetime.

Our immediate goals are to combine the CS anode with $6 \mu \mathrm{m}$ pore MCP's for high resolution imaging tests, and with the new generation silicon small pore MCP's [8]. Larger anodes $(25 \mathrm{~mm})$ are being designed to accommodate standard detector format sizes. New low power amplifier chips with the preamplifier, shaper, discriminators and signal latching are in the design phase. These, combined with high speed CMOS ADC's and a centroid algorithm performed by high speed DSP chips should enable the full bandwidth of the CS anode to be demonstrated in the near future.

\section{ACKNOWLEDGMENTS}

We would like to thank Dr. J. Millaud for the preamplifier chips and advice on the electronics. We also acknowledge the contributions of Mr. J. Zaninovich to the electronics design.

\section{REFERENCES}

[1] O.H.W. Siegmund, "Amplifying and position sensitive detectors," in "Methods of vacuum ultraviolet physics" Chapter III, 2nd edition, editors J.A.R. Samson and D.L. Ederer, Academic Press, 1998.

[2] O.H.W. Siegmund, J. Zaninovich, A.S. Tremsin, J.S. Hull, "Cross strip anodes for MCP imaging detectors", Proc. SPIE, vol. 3445, pp.397-406, July 1998.

[3] O.H.W. Siegmund, P. Jelinsky, S. Jelinsky, J. Stock, J. Hull, D. Doliber, J. Zaninovich, A.S. Tremsin \& K. Kromer, "High resolution cross delay line detectors for the GALEX mission", Proc. SPIE, vol. 3765. pp. 429-440, 1999.

[4] W. E. Hearn, I. Kipnis. H. von der Lippe \& T. C. Collins, LBL Microelectronics Group, Internal Report, Aug. 1993.

[5] A.S. Tremsin, O.H.W. Siegmund, "Measurements of metric nonlinearities of MCP-based lobster eye x-ray telescope optics by Moire interferometry", Proc. SPIE, vol 3356, pp.866-875, 1998.

[6] .J.S. Lapington, \& B. Sanderson, "Microchannel plate limited resolution with the vernier anode," Proc. SPIE, vol. 4139, preprint, 2000.

[7] J.V. Vallerga, O.H.W. Siegmund, J. Dalcolmo, \& P. Jelinsky, "A high resolution (<10 micron) photon counting $\&$ analog intensified CCD”, Proc. SPIE, vol. 3019, pp.156167, 1997.

[8] O. H.W. Siegmund, A. S. Tremsin, J. V. Vallerga, C. P. Beetz, R.W. Boerstler \& D. Winn, "Silicon Microchannel Plates: Initial Results for Photon Counting Detectors" Proc. SPIE, vol. 4140, preprint, 2000 